\title{
Numerical prediction of the ballistic performance of hygrothermally aged CFRP laminates using a multi-scale modelling approach
}

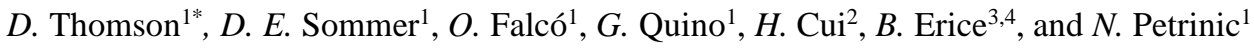 \\ ${ }^{1}$ Department of Engineering Science, University of Oxford, Oxford, United Kingdom \\ ${ }^{2}$ School of Civil Aviation, Northwestern Polytechnical University, Suzhou, China \\ ${ }^{3}$ Department of Mechanics and Industrial Production, Mondragon Unibertsitatea, Mondragon, Spain \\ ${ }^{4}$ IKERBASQUE, Basque Foundation for Science, Bilbao, Spain
}

\begin{abstract}
A multi-scale modelling strategy is proposed to investigate the potential effects of hygrothermal ageing on the ballistic performance of CFRP laminates. At the micro-scale, damage evolution due to cyclic hygrothermal loads is simulated on a representative microstructure using an adapted fatigue damage law. Then, the results of the micro-mechanical analysis are used to measure the predicted transverse crack density and calibrate a meso-scale material model with the effects of micro-scale damage. Finally, a meso-scale impact model is generated with seeded transverse cracks at the predicted crack density and the calibrated material properties from the homogenisation step, which allows for the performance of the laminate to be compared at different stages of hygrothermal ageing damage.
\end{abstract}

\section{Background and introduction}

Due to the time and cost required for experimental characterisation of environmental ageing effects in CFRP laminates, the possibility of using numerical modelling to predict this behaviour is investigated alongside an ongoing experimental campaign that will provide data for evaluation of the proposed modelling strategy.

Cyclic loading and environmental conditions during the lifetime of CFRP components can have significant negative effects on their material properties and performance. Previous studies on mechanical fatigue, hygroscopic absorption, temperature and thermal cycling have shown reductions in stiffness, strength, fracture toughness properties can be caused by a combination of chemical changes in the constituents and different types of damage, including micro-damage at the fibre-matrix interfaces (and/or toughening particles) as well as larger scale matrix cracking and/or delamination $[1,2]$.

Therefore, due to the combination of micro- and meso-scale mechanisms, physically sound numerical modelling of these phenomena requires consideration of the different length scales. The following multi-scale FEM modelling approach is proposed:

- Micro-scale modelling to predict the effects of the hygrothermal cycling.

* Corresponding author: daniel.thomson@eng.ox.ac.uk 
- Homogenisation of the micro-scale damage and calibration of meso-scale models.

- Meso-scale modelling of the structural and impact performance after ageing.

Using this proposed multi-scale modelling approach, the material performance is evaluated at different stages of the hygrothermal ageing process: first, at a dry, pristine, or undamaged, state before ageing; then, at a point of widespread micro-damage, before the onset of meso-scale cracking; and, last, at different stages of increasing meso-scale matrix cracking.

The material performance at each stage is evaluated through dynamic three-point bending (D3PB) simulations at different velocities to investigate the effects of ageing on the ballistic limit and impact energy dissipation. Finally, these predictions will be compared against experimental results when they become available at the end of an ongoing experimental campaign, in order to verify the accuracy and investigate current limitations and potential improvements of the proposed multi-scale modelling strategy.

\section{Micro-scale}

At the micro-scale, the response of a detailed representative microstructure under hygrothermal cyclic loads, is modelled with temperature- and moisture-dependent material properties, continuum damage mechanics and fatigue damage laws [3] to predict the state of damage in the material.

\subsection{Model set-up}

For the micro-mechanical model to give accurate predictions, three things are needed: a representative description of the material's microstructure; accurate models for the material behaviour of the different constituents under the considered environmental conditions; and a realistic representation of the applied loads.

\subsubsection{Representative microstructure}

A $2 \mathrm{D}$ representative section of $0.125 \times 1 \mathrm{~mm}$ was generated by a random fibre placement algorithm using the Digimat FE software from e-Xstream. The length of the model was necessary to capture the evolution of the crack density under cyclic loading. Plies above and below were represented with homogenised material properties to simulate a 0/90 lay-up, Figure 1. The generated microstructure was imported to Abaqus CAE and meshed using 2D plane strain elements with cohesive elements used for fibre-matrix interface. The homogenised plies above and below were joined using the built-in cohesive interaction with coupled frictional behaviour.

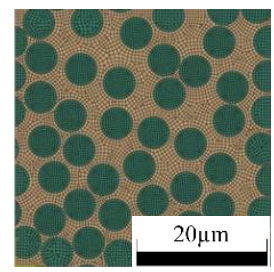

(a)

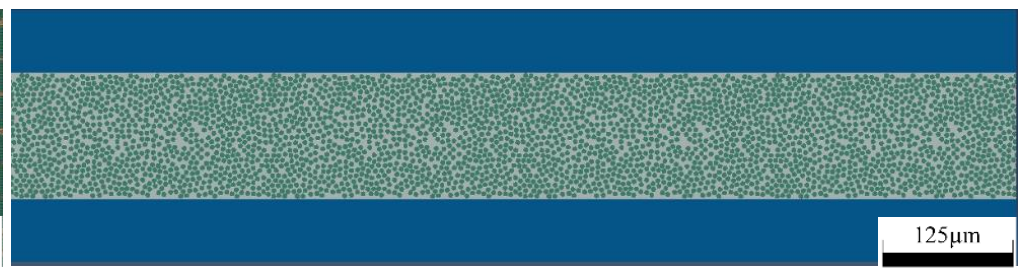

(b)

Fig. 1. Generated microstructure using a random fibre placement algorithm (a) and entire $1 \mathrm{~mm}$ length model including homogenised half-plies above and below (b). 


\subsubsection{Constituent properties}

The IM7 carbon fibres were given orthotropic elastic material properties and orthotropic thermal expansion coefficients taken from the literature [4,5], while the moisture content and cyclic loading were assumed to have no effect on their behaviour. The 8552 toughened epoxy resin was modelled using the built-in concrete damaged plasticity model in Abaqus/Standard, calibrated from experimental data in the literature, and an isotropic thermal expansion coefficient $[6,7]$. The stiffness, strength, and fracture energy were made temperature and moisture-dependent by giving tabulated field-dependent properties calibrated from similar epoxy resins in the literature $[2,8]$. The fibre-matrix interface was modelled using a bilinear cohesive law calibrated to match the macroscopic response of the composite under static, room temperature, dry conditions, and the effects of temperature and moisture content were assumed to be proportional to those in the matrix. Finally, the longitudinal plies above and below were given homogenised orthotropic elastic properties and hygroscopic and thermal expansion coefficients for IM7/8552. Key material parameters are summarized in Table 1 below, the coefficient of hygroscopic expansion, $\beta_{H}$, is given in units of strain per $1 \%$ change in relative humidity, $\varphi^{0-1}$.

Table 1. Summary of key constituent properties obtained from [2,4-8].

\begin{tabular}{ccccccc}
\hline Fibre & $E_{1}(\mathrm{GPa})$ & $E_{2}(\mathrm{GPa})$ & $G_{23}(\mathrm{GPa})$ & $v_{12}$ & $\alpha_{T 1}\left(\mathrm{~K}^{-1}\right)$ & $\alpha_{T 2}\left(\mathrm{~K}^{-1}\right)$ \\
\hline IM7 & 273 & 15 & 5.5 & 0.35 & $-6 \mathrm{e}-7$ & $6 \mathrm{e}-6$ \\
\hline \hline Matrix & $E(\mathrm{GPa})$ & $v$ & $\sigma_{t}(\mathrm{MPa})$ & $G_{C}\left(\mathrm{~J} / \mathrm{m}^{2}\right)$ & $\alpha_{T}\left(\mathrm{~K}^{-1}\right)$ & $\beta_{H}\left(\varphi^{0-1}\right)$ \\
\hline 8552 & 5 & 0.35 & 121 & 100 & $5 \mathrm{e}-6$ & $6 \mathrm{e}-5$ \\
\hline
\end{tabular}

\subsubsection{Hygrothermal loads}

Thermal loads were applied by controlling the temperature field over time. The changes in temperature were assumed slow enough to produce a homogeneous temperature across the entire model and the thermal loads were introduced as a result of the mismatch in thermal expansion coefficients. The same was done for the relative moisture content in the matrix by using a user-defined field variable to control the increase in humidity up to saturation. The effect of thermal cycling on the matrix and interfaces was introduced through a second userdefined field variable to represent the accumulation of fatigue damage over a number of cycles as a function of the maximum principal strain and the load ratio [3]:

$$
\Delta D / \Delta N=\alpha\left(\varepsilon_{e f f}\right)^{\beta}
$$

where $\Delta D / \Delta N$ is the increase in fatigue damage, $D$, over a number of cycles, $N$; $\varepsilon_{\text {eff }}$ is the effective fatigue strain, including a correction for the load ratio effect; and $\alpha$ and $\beta$ are constants that define the damage evolution.

The simulation was started at the curing temperature of $150^{\circ} \mathrm{C}$, then cooled down to room temperature to generate the internal thermal stresses. Next, the moisture content was linearly increased up to the saturation limit through the user-defined field, which caused some relaxation of the thermal stresses due to the hygroscopic expansion. Finally, the thermal cycling, from -55 to $90 \mathrm{C}$, was applied assuming constant moisture content throughout the step. The damage due to cyclic loading was modelled by further reducing the temperature to the lowest state in the cycle, which introduced the greatest internal strains, and incrementally adding to the number of cycles, $N$, and therefore the damage, $D$, within the user-defined field described above. 


\subsection{Results}

The first signs of damage appeared in the form of interface damage between fibre and matrix, growing in magnitude and in number until most interfaces were damaged in the vertical direction on both sides of the fibre, Figure 2 (a). After this point, damage eventually begins to appear in the matrix and propagates in the form of transverse cracks. The formation of these matrix cracks unloads the nearby material, reducing the likelihood of matrix cracks within a certain distance, and redistributes the tensile load to the undamaged material above and below the crack tips. This stress redistribution and stress concentrations cause the fatigue damage to accelerate at the crack tips, until the crack eventually propagates across the entire ply thickness. At this point, depending on the laminate architecture, damage may continue to propagate in the form of delamination. However, for the 0/90 lay-up studied here, the lack of interlaminar shear stress to drive this damage mode resulted in no significant delamination. Multiple cracks may start to form at different points in the material, growing in number with the number of cycles. With adequately calibrated fatigue parameters, the crack density in the investigated laminate and loading conditions reached approximately $2 \times$ the ply thickness of $0.125 \mathrm{~mm}$ after approximately 2000 cycles, Figure 2 (b), in line with the thermal cycling results in [1].

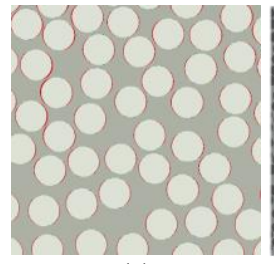

(a)

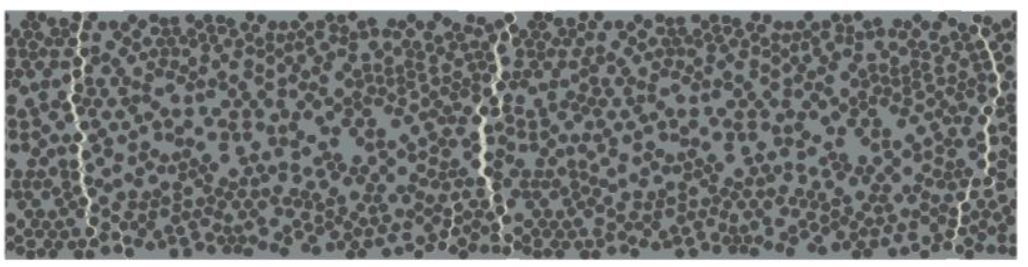

(b)

Fig. 2. Initial fibre-matrix interface damage (a) and transverse matrix cracks (b).

\section{Meso-scale}

Finally, the mechanical and ballistic performance of the aged material was predicted using a high-fidelity fibre-aligned mesh with constitutive behaviour calibrated from the micromechanical model results and seeded transverse matrix cracks. The investigated test case is a dynamic three-point bending (D3PB) test on a $15 \times 80 \mathrm{~mm}\left[(0 / 90)_{5} / 90\right]_{\mathrm{S}} \mathrm{IM} 7 / 9552$ laminate with a 0.125 nominal ply thickness, which will be performed as part of an ongoing experimental campaign to study hygrothermal ageing effects.

\subsection{Model set-up}

The material behaviour at the meso-scale was modelled using a pressure- and rate-dependent plasticity and damage model in LS-DYNA [9]. The effects of fibre-matrix interface damage on the homogenised material properties were modelled as a reduction in transverse and shear moduli proportional to the reduction in stiffness of the micro-scale model. Next, the larger matrix cracks, which must be accounted for separately from the micro-scale interface damage, were quantified by measuring the crack density. The laminate was meshed using ply-by-ply fibre-aligned elements, Figure 3, using under-integrated solid elements with hourglass control. The interface between adjacent plies was modelled using the built-in cohesive contact formulation in LS-DYNA, tiebreak contact, with interface properties calibrated for IM7/8552. Finally, different models were generated with different number of transverse cracks by detaching elements along the fibre direction, up to the crack density 
predicted in the micro-mechanical model, Figure 3 (c). The $10 \mathrm{~mm}$ diameter steel projectile and the two supports with a span length of $65 \mathrm{~mm}$ were modelled as rigid bodies.

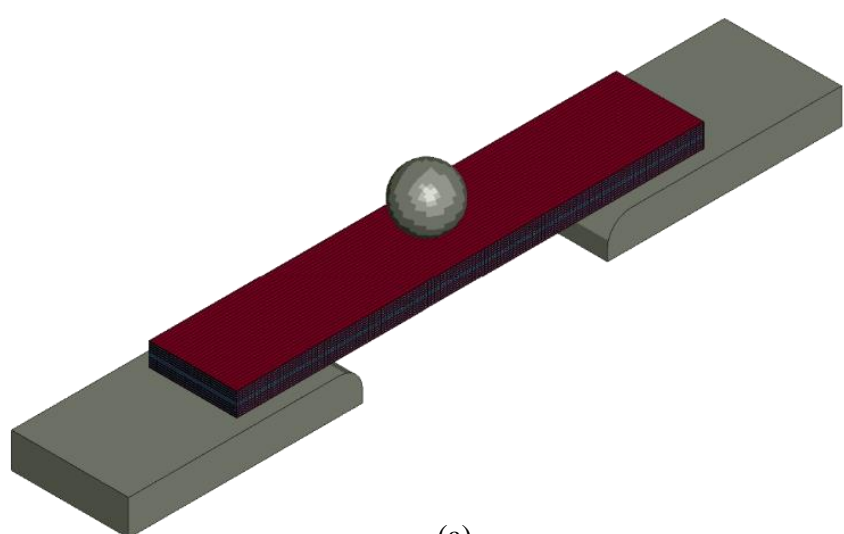

(a)

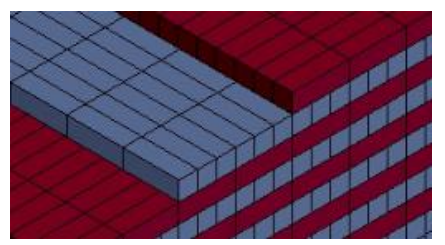

(b)

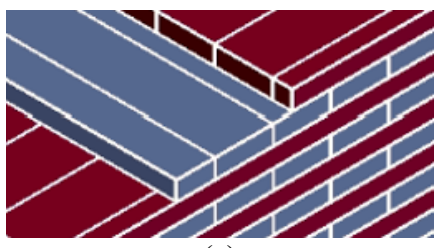

(c)

Fig. 3. Finite element model used for the dynamic three-point bending simulations (a); fibre-aligned ply-by-ply element mesh (b); and detached rows of elements along the fibre direction to simulate the transverse cracks form hygrothermal ageing damage (c).

\subsection{Results}

From simulations on the pristine material, an impact velocity of $75 \mathrm{~m} / \mathrm{s}$ was found to be close to ballistic limit. This was selected as the reference for all other models to compare the effects of different amounts of hygrothermal ageing on the ballistic performance of the cross-ply IM7/8552 laminate.

Results of the meso-scale simulations are shown in Figure 4 for three different stages of damage. First, the pristine material with no hygrothermal ageing. Then an intermediate damage state with random transverse cracks inserted across all plies along the length of the ply with an average relative crack density of approximately $\bar{\rho}=\rho t_{N}=0.25$, where $\rho=1$ / $l$ is the crack density calculated from the average distance between cracks and $t_{N}$ is the ply thickness. Finally, a fully aged state, with a normalised crack density of $\bar{\rho}=0.5$.

The results show a $15 \%$ reduction in peak force at the intermediate damage state and $20 \%$ for the fully aged material, which may seem relatively little compared to the extent of cracking in the material. However, as observed in the micro-mechanical model, the delamination interfaces, which are one of the principal mechanisms of energy dissipation under impact, remained relatively intact in the 0/90 laminate during hygrothermal ageing. Similar observations can be made for fatigue loading of 0/90 laminates in [10]. Therefore, the remaining inter-ply strength may give the laminate the predicted residual performance under impact even after significant matrix cracking. In the case of quasi-isotropic or multidirectional laminates, inter-ply shear stresses during the cyclic loading would likely introduce significant delamination damage, which may more severely affect impact performance. Therefore, if these results can be confirmed by the ongoing experimental campaign, it may indicate that 0/90 lay-ups may be more well suited for applications with significant hygrothermal or other fatigue loading conditions. 


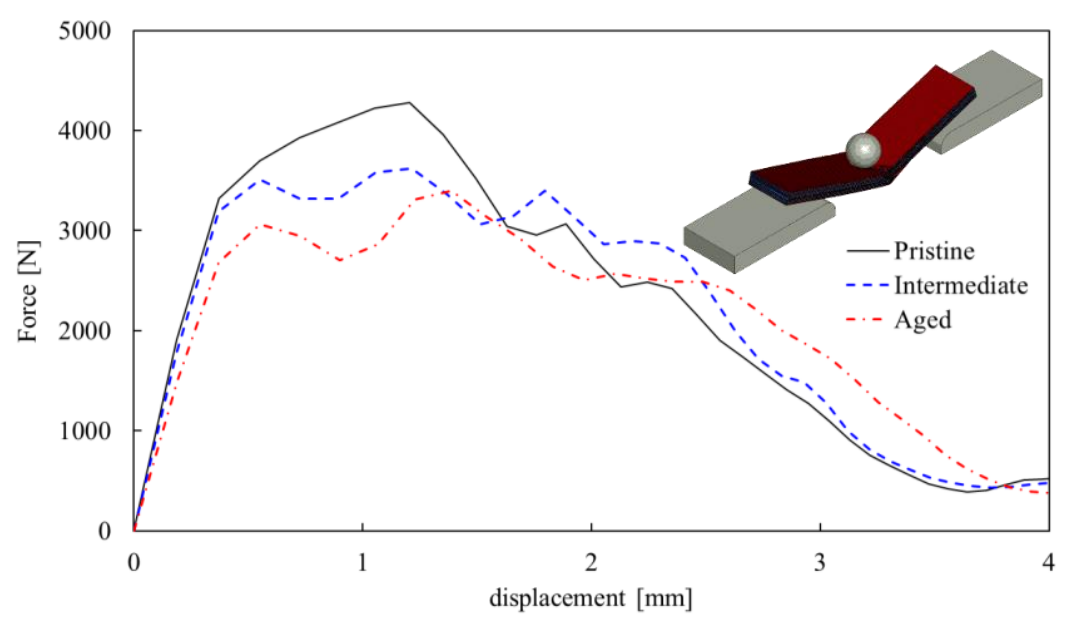

Fig. 4. Results of D3PB simulations on the IM7/8552 cross-ply beam at an impact velocity of $75 \mathrm{~m} / \mathrm{s}$. The solid black line represents the pristine material with no hygrothermal ageing; the dashed blue line represents an intermediate state of damage; and the dotted red line represents the fully aged material.

\section{Conclusions}

A multi-scale modelling study has been performed on a 0/90 IM7/8552 laminate under hygrothermal cyclic loading. A micro-mechanical model was used to predict the state of damage in the material, including fibre-matrix interface damage and transverse matrix cracks, after a number of load cycles. Based on these results, a meso-scale material model was calibrated and a dynamic three-point bending test was simulated with a fibre-aligned mesh and seeded matrix cracks at the predicted crack density. The results show the predicted effects of hygrothermal ageing on the ballistic performance of the beam, which will be verified at the conclusion of an ongoing experimental campaign.

The authors would like to acknowledge Rolls-Royce plc, for their continuing support through the Solid Mechanics University Technology Centre at the University of Oxford.

\section{References}

1. C. Lüders, M. Sinapius, J. Compos. Mater., 53, 20 (2019).

2. G. Quino, J. El Yagoubi G. Lubineau, Polym. Degrad. Stab., 109 (2014).

3. K.B. Katnam, A.D. Crocombe, H. Khoramishad, I.A. Ashcroft, J. Adhes., 86, 3 (2010).

4. Hexcel, "HexTow ${ }^{\circledR}$ IM7 Carbon Fiber," (2018).

5. R. Kulkarni, O. Ochoa, J. Compos. Mater., 40, 8 (2006).

6. Hexcel, "HexPly 8552 Data Sheet," (2017).

7. F. Naya, C. González, C.S. Lopes, S. Van der Veen, F. Pons, Compos. Part A Appl. Sci. Manuf., 92, (2017).

8. S. Alessi, D. Conduruta, G. Pitarresi, C. Dispenza, G. Spadaro, Polym. Degrad. Stab., 96, 4 (2011).

9. D. Thomson, B. Erice, H. Cui, J. Hoffmann, J. Wiegand, N. Petrinic, Comp. Struct., 184 (2018).

10. J. Llobet, P. Maimí, Y. Essa, F. Martin de la Escalera, Int. J. Fatigue, 119, (2019). 